\title{
Contrast and Asymmetries in Inventories
}

\author{
B. Elan Dresher \\ University of Toronto
}

\begin{abstract}
One source of asymmetry in phonology derives from the contrastive function of features. I assume that feature specifications are driven by considerations of contrast. In order to distinguish between contrastive and redundant specifications, it is necessary to specify the relative scope, or ordering, of each feature. This ordering determines a contrastive hierarchy. Different contrastive hierarchies create different asymmetries in inventories, and thus different expectations as to how the system will pattern. I will briefly review the history of the contrastive hierarchy, and illustrate it with respect to synchronic and diachronic patterns in the vowel systems of the Manchu languages.
\end{abstract}

\section{Introduction}

There are a number of sources of asymmetry in phonology. One has to do with the different possibilities accorded to phonological heads as opposed to dependents (Dresher and van der Hulst 1998). In this paper I would like to focus on asymmetry that derives from the contrastive function of features. I assume that feature specifications are, at least to some degree, driven by considerations of contrast. A segment must be sufficiently specified so that it is differentiated from other segments in the inventory: the greater the number of contrasts, the more specifications will be required. Specifications that are required to draw a contrast between segments are contrastive with respect to those segments; specifications that are not contrastive are redundant. Phonologists have debated to what extent redundant specifications play a role in phonology. But the debate has necessarily been inconclusive, because of a lack 


\section{B. Elan Dresher}

of a clear notion as to what makes a specification contrastive or redundant in any given instance.

I will argue that in any inventory characterized by more than one distinctive feature it is necessary to specify the relative scope of each feature by means of a contrastive hierarchy. I will begin with some general observations about the logic of contrast. Then I will briefly look at the history of the notion of the contrastive hierarchy in phonological theory. Finally, I will illustrate the workings of the contrastive hierarchy with respect to synchronic and diachronic patterns in the vowel systems of the Manchu languages.

\section{The Logic of Contrast}

I'd like to begin with some general observations about the logic of contrast. I will employ non-linguistic items at first, so that it is clear that we are talking about contrast in a general way, and not about anything unique to phonology or morphology, or even language.

To begin, consider a simple inventory made up of the two items in (1).

(1) Inventory 1: Full specification

\begin{tabular}{lll}
\hline & A. $\boldsymbol{~}$ & B. $\mathrm{O}$ \\
\hline Shape & square & round \\
Size & small & large \\
Colour & shaded & clear \\
\hline
\end{tabular}

In (1) there are two contrasting figures. If these are the only items in the inventory, we may take them to be primitive elements, not further decomposable into constituent properties. Nevertheless, it is human nature to attempt to do just that, to try to identify characteristic distinguishing properties, or features. We observe that the two items differ on a number of dimensions:

a) Shape: Item $\mathrm{A}$ is square, $\mathrm{B}$ is a circle.

b) Size: Item A is smaller, B is larger.

c) Colour: Item A is shaded, $\mathrm{B}$ is clear.

Given just A and B, we have no way to tell whether all these features are essential to the identity of the item, or if any is more important than the others. It could be that one of these features is criterial, or relevant or contrastive, and the others are simply 
along for the ride, or redundant. It is also possible that some of these features form a gestalt that cannot be unpacked. Without some further information about how the members of this inventory behave, we can't tell.

Now let us look at Inventory 2, as shown in (2). Here we have three contrasting members: a small square (A), a large square (B), and a large circle (C). These members can be characterized by two features, shape and size. They are thus distinctive features, because they play a role in distinguishing between members.

(2) Inventory 2: Full specification

\begin{tabular}{llll}
\hline & A. $\square$ & B. $\square$ & c. $\bigcirc$ \\
\hline Shape & square & square & round \\
Size & small & large & large \\
\hline
\end{tabular}

There is no doubt that each feature is distinctive somewhere in the inventory: shape alone distinguishes $\mathrm{B}$ from $\mathrm{C}$, and size alone distinguishes $\mathrm{A}$ from $\mathrm{B}$. But are both features contrastive with respect to each member of the inventory? In other words, do we need to specify both features for each member in order to uniquely distinguish it from the others? The answer is clearly no. In Inventory 2, A is the only member that is small, and C is the only member that is round. It follows that the feature [small] is sufficient to characterize A, and [round] immediately picks out C. Therefore, it ought to follow, so one might think, that a more economical specification would be as in (3).

(3) Inventory 2: Contrastive specification (incorrect!)

\begin{tabular}{llll}
\hline & A. $\square$ & B. $\square$ & C. $\bigcirc$ \\
\hline $\begin{array}{l}\text { Shape } \\
\text { Size }\end{array}$ & small & $\begin{array}{l}\text { square } \\
\text { large }\end{array}$ & round \\
\hline
\end{tabular}

Let's say that a contrastive specification is one that uses only as many specifications as are required to distinguish each member from every other member. Is (3) a contrastive specification? Let's see if every member is minimally distinguished from every other one.

There are three pairwise comparisons to make. First, A and B are distinguished by size: $\mathrm{A}$ is [small] and $\mathrm{B}$ is [large]. $\mathrm{B}$ and $\mathrm{C}$ have a minimal contrast based on shape, [square] vs. [round]. 


\section{B. Elan Dresher}

But consider now A and C: the first is contrastively [small], and the second is contrastively [round]. Do these specifications allow us to draw a contrast between them? The answer is no. Their specifications look different, but they are not distinct. Indeed, the relation between $\mathrm{A}$ and $\mathrm{C}$ with respect to shape is like that between $\mathrm{A}$ and B: one is specified, the other is not. But A and B have the same shape. There is nothing in the chart in (3) that tells us that in this case the unspecified shape is not the same as the specified one. Similar considerations hold for size. We can, of course, write a rule that no specification for shape results in [square] and no specification for size results in [large]. Without applying at least one of these rules, we cannot tell if $\mathrm{A}$ and $\mathrm{C}$ are in contrast or not. But then we have failed in our attempt to represent all the relevant contrasts in the chart.

What has gone wrong here? In fact, the chart in (3) results from a misconstrual of our original observations about the inventory. When I wrote above that "A is the only member that is small," what I had in mind was that once we made a size contrast between A on one side ([small]) and B and C on the other ([large]), there is no need to further specify A for shape. The relevant contrasts can be pictured as in (4a). And when I wrote, " $\mathrm{C}$ is the only member that is round," I had in mind a picture such as (4b), where once C is specified [round] and A and B [square], there is no need to further specify $\mathrm{C}$.

(4) Inventory 2: Two ways of viewing the contrasts

a. A is the only small member

\begin{tabular}{c|c:c}
\hline small & \multicolumn{2}{|c}{ large } \\
A. $\square$ & B. $\square$ & C. $\bigcirc$ \\
& square & round \\
\hline
\end{tabular}

b. $\quad \mathrm{C}$ is the only round member

\begin{tabular}{c|c:c} 
square & \multicolumn{2}{|c}{ round } \\
A. $\square$ & B. $\square$ & C. $\bigcirc$ \\
small & large & \\
\hline
\end{tabular}

The diagram in (4a) corresponds to the feature specifications in (5a), and (4b) corresponds to $(5 \mathrm{~b})$.

(5) Inventory 2: Two sets of contrastive specifications

\begin{tabular}{|c|c|c|c|c|c|c|}
\hline \multicolumn{3}{|c|}{ According to (4a) } & & \multicolumn{3}{|c|}{ b. According to (4b) } \\
\hline A. $\square$ & B. & $\mathrm{C}$ & & А. $\square$ & B. & C. \\
\hline small & $\begin{array}{l}\text { square } \\
\text { large }\end{array}$ & $\begin{array}{l}\text { round } \\
\text { large }\end{array}$ & $\begin{array}{l}\text { Shape } \\
\text { Size }\end{array}$ & $\begin{array}{l}\text { square } \\
\text { small }\end{array}$ & $\begin{array}{l}\text { square } \\
\text { large }\end{array}$ & round \\
\hline
\end{tabular}


Contrast and Asymmetries in Inventories 5

Diagrams (4a) and (4b) and their corresponding feature charts in (5a) and (5b) represent two distinct ways of "cutting up" the inventory. In (4a), the main contrast is size. We first divide up all the members of the inventory into two sets, one [large] and one [small]. Then we make a shape distinction in the [large] set. The [small] set has only one member, so no further contrasts in that set could be contrastive. Therefore, member A is contrastively specified for size, but only redundantly specified for shape. However, B and C are contrastively specified for both size and shape.

In (4b) the inventory is cut differently. Here, the first cut is by shape, and the second one is by size. The difference between (4a) and (4b) is in the ordering, or scope, of the features. In (4a), the size feature is ordered before the shape feature (i.e., has wider scope), and in (4b) size is ordered after shape (has narrower scope). Another way to represent these cuts is by tree diagrams, as in (6a) and (6b). The notation A > B means "A is ordered before B," or "A takes wider scope than B."

(6) Inventory 2: Tree diagrams of contrastive cuts

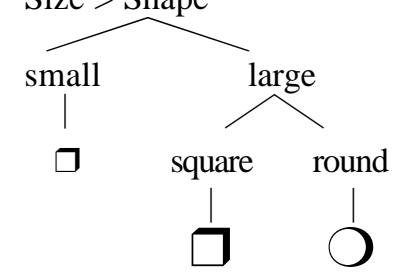

b. Shape $>$ Size

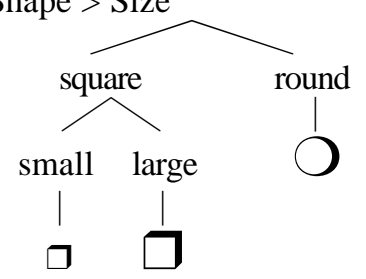

Thus, the observations that $\mathrm{A}$ is the only small member and $\mathrm{C}$ is the only round member are correct, but in terms of contrastive force they derive from two different ways of cutting up the inventory. The ultra-minimal specification in (3) results from trying to put together two observations that derive from incompatible ways of cutting up the inventory. For this reason, it fails to adequately contrast $\mathrm{A}$ and $\mathrm{C}$.

One reason that (3) may have looked correct is that, unlike (5a) and (5b), it contains no logically redundant features. We can define logical redundancy as in (7).

(7) Logical redundancy

If $\Phi$ is the set of feature specifications of a member, $M$, then the feature specification $[\mathrm{F}]$ is logically redundant iff it is predictable from the other specifications in $\Phi$. 


\section{B. Elan Dresher}

A feature that is logically redundant is predictable from the other features. In Inventory 2, the feature [square] is predictable from [small], and [large] is predictable from [round]. However, logical redundancy is not sufficient to make a feature redundant in any particular contrastive hierarchy.

To see this very clearly, consider again Inventory 1, with only two contrasting members. Here, every feature is predictable given the other two. Indeed, any two features are predictable given the other one. Thus, all three features are logically redundant, but they can't all be omitted! Rather, logical redundancy indicates at most potential redundancy. Removing all logically redundant features often results in incoherence.

Another reason that we may not notice immediately that (3) fails to provide a set of adequate contrasts is that, when presented with a set of contrasting items, there is a tendency to concentrate on minimal pairs. A minimal pair is a pair of set members that differ from each other with respect to only one feature. It makes sense to focus on minimal pairs, because minimal pairs reveal the contrasting features in the purest way. It appears that many phonologists have implicitly or explicitly adopted a version of what we can call the Minimal Pairs Test.

(8) The Minimal Pairs Test (incorrect!)

To ensure that all members of an inventory are in contrast with each other, it is sufficient to specify contrasts between all potential minimal pairs.

The Minimal Pairs Test fails to ensure that all members of an inventory are in contrast. As with redundancy, minimal pairs with respect to full specification of distinctive features (weak minimal pairs) are only potentially minimal pairs given a particular contrastive hierarchy (strong minimal pairs). Thus, the large square, member B in Inventory 2, is weakly a minimal pair with both members A and C. But as the diagrams in (6) make clear, it forms a strong minimal pair with only one of these in any hierarchy.

There is a third logical possibility for how to characterize the contrasts in this inventory. Suppose that size and shape were equal in scope, so that none takes precedence over the other. Then we have to imagine the two ways of cutting up the inventory superimposed on each other, resulting in true cross-classification. This results in the full specification of (2), not the underspecification of (3). For in this approach, both features of every member are contrastive. In true cross-classification, we evaluate each feature independently from every other one. Looking only at shape, 
A and B are [square] and C is [round]. Looking only at size, A is [small] and B and C are [large]. On this model, member $\mathrm{B}$ is equidistant from $\mathrm{A}$ and $\mathrm{C}$, as shown schematically in (9).

(9) Size $=$ Shape: $\mathrm{B}$ is equidistant from $\mathrm{A}$ and $\mathrm{C}$

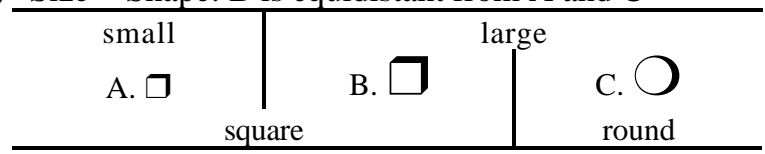

What we conclude from the above discussion is that, when presented with an inventory and list of distinctive features, there is not necessarily a unique solution to what the contrastive specifications are. In any system of sufficient complexity, there will be a number of possible ways of structuring the system, depending on the ordering of relevant features. If ordering is completely free, then for $n$ distinctive features there are $n$ ! possible orderings.

Not every ordering leads to a unique set of contrastive specifications. In general, the more symmetric an inventory is, the more the different feature orderings converge on the same specifications. That is, a symmetric inventory more closely approaches the appearance of cross-classification. The more asymmetric the inventory, the more dramatic the effect of different orderings. Of course, the apparent symmetry of an inventory is itself partially a function of the way the features are ordered.

\section{The contrastive hierarchy via the Successive Division Algorithm}

Our discussion of domains of contrast and the relative scope of features suggests that a natural way of determining contrast is by splitting the inventory by means of successive divisions, governed by an ordering of features. An algorithm corresponding to this idea, the Successive Division Algorithm (SDA), is given in (10):

(10) Successive Division Algorithm (SDA)

a. In the initial state, all tokens in inventory, I, are assumed to be variants of a single member. Set $\mathrm{I}=\mathrm{S}$, the set of all members.

b. i) If $\mathrm{S}$ is found to have more than one member, proceed to (c). 
8 B. Elan Dresher

ii) Otherwise, stop. If a member, $\mathrm{M}$, has not been designated contrastive with respect to a feature, $\mathrm{G}$, then $\mathrm{G}$ is redundant for M.

c. Select a new $n$-ary feature, $\mathrm{F}$, from the set of distinctive features. ${ }^{1} \mathrm{~F}$ splits members of the input set, $\mathrm{S}$, into $n$ sets, $\mathrm{F}_{1}-\mathrm{F}_{n}$, depending on what value of $F$ is true of each member of $S$.

d. i) If all but one of $\mathrm{F}_{1}-\mathrm{F}_{n}$ is empty, then loop back to (c). ${ }^{2}$

ii) Otherwise, $\mathrm{F}$ is contrastive for all members of $\mathrm{S}$.

e. For each set $\mathrm{F}_{i}$, loop back to (b), replacing $\mathrm{S}$ by $\mathrm{F}_{i}$.

The algorithm in (10) is a very general formulation for defining contrastiveness and redundancy for members of an inventory. It can apply to $n$-ary features and it does not tie contrastiveness and redundancy to how a member is specified. In practice, though, the most natural assumption to make is that all members that are contrastive for a feature, $\mathrm{F}$, receive specifications for $\mathrm{F}$, and members that are redundant do not.

\section{Binary (equipollent) vs. unary (privative) features}

Another source of asymmetry in phonology comes from the nature of the features themselves. So far I have assumed binary features where the two values are equal (equipollent features). But this may not be what linguistic features are like. It could be that one value is marked and the other is unmarked. We could take markedness literally, so that only the marked value is marked, and the unmarked value is simply the absence of the marked one. This is a privative feature system

To make an equipollent contrast between round and square, it is enough to write [round] $\sim$ [square], or [+round] $\sim$ [-round], or [-square] $\sim$ [+square]. To make a privative contrast, we have to decide which is the marked feature. For the sake of discussion, let us set up the following privative features as in (11).

(11) Privative features for size, shape, and colour

\begin{tabular}{lccc}
\hline & Size & Shape & Colour \\
\hline marked & large & round & shaded \\
unmarked & $($ small $)$ & (square) & (clear) \\
\hline
\end{tabular}


In (11) the features in parentheses are defaults that do not actually appear in contrastive specifications. They are there only to remind us what the absence of a value entails. The only actual marks are the marked values.

Fully specifying all distinctive features for Inventory 2 now results in (12).

(12) Inventory 2: Full specification (privative features)

\begin{tabular}{lccc}
\hline & A. $\square$ & B. $\square$ & c. $\bigcirc$ \\
\hline $\begin{array}{l}\text { Shape } \\
\text { Size }\end{array}$ & large & $\begin{array}{l}\text { round } \\
\text { large }\end{array}$ \\
\hline
\end{tabular}

Note that the specifications of A and B are already minimal, so only the specifications of $\mathrm{C}$ can be affected by ordering. If size is the first feature, then we observed (6a) that $A$ is the only member that is small. Using equipollent features, we marked A [small] and B and C [large]. But now the former is not a specification, so we leave A unmarked and mark B and C as [large]. The next contrast is between $\mathrm{B}$ and $\mathrm{C}$ on the basis of shape, and we mark $\mathrm{C}$ as [round]. Now we have the same specifications as in (12). Proceeding in the other order, we first mark $\mathrm{C}$ [round] in contrast to A and $\mathrm{B}$, which are unmarked. We then draw a contrast between $\mathrm{A}$ and $\mathrm{B}$ by marking $\mathrm{B}$ [large]. This time, $\mathrm{C}$ is specified only as [round].

The effect of feature ordering is reduced with privative features as compared with equipollent features. For similar reasons, the relationship between contrastiveness and specification is more transparent with equipollent than with privative features. This is because in an equipollent system, if a member, $\mathrm{M}$, is specified for a feature, $\mathrm{F}$, then $\mathrm{F}$ is contrastive for $\mathrm{M}$. Conversely, if $\mathrm{M}$ is unspecified for $\mathrm{F}$, then $\mathrm{F}$ is redundant for $\mathrm{M}$. In a privative system, specification indicates contrastiveness, as in an equipollent system. However, if $\mathrm{M}$ is unspecified for $\mathrm{F}$, then either i) $\mathrm{F}$ is redundant for $\mathrm{M}$, or ii) $\mathrm{M}$ is unmarked for $\mathrm{F}$ (or both (i) and (ii) are true).

\section{A very brief history of the contrastive hierarchy in phonology}

Since Saussure it has been commonly stated that contrast is central to linguistic theory, but there has been relatively little discussion of how contrastiveness is to be assigned in general. With a few notable exceptions, there is almost nothing in the phonological literature on the notion of a contrastive hierarchy, much less that it is 


\section{B. Elan Dresher}

central to establishing contrast. Nevertheless, a hierarchy has been implicit in at least a partial way in the practice of phonologists.

One place to see this is in the way tables of segmental inventories are arranged. Compare, for example, the tables of Siglitun (13) and Kolokuma Ijo (14). I present them as they are given in the sources (with some changes to the phonetic symbols but not to the arrangement). Note in particular the different placements of $/ 1 /$ and $/ \mathrm{j} /$ in these charts. The chart of Ijo expresses a hierarchy whereby the feature [continuant] has wider scope than such features as [sonorant] and [voiced], and [lateral] has wider scope than [nasal]. The Siglitun chart is not as overtly hierarchical, but it is clear that the feature [lateral] has very narrow scope, confined to making distinctions among apicals, whereas [nasal] is higher in the hierarchy. Apart from the nasals, the other sonorants are not set apart in Siglitun, suggesting that the feature [sonorant] is lower in the hierarchy than in Ijo

(13) Siglitun consonants (Dorais 1990:70) ${ }^{3}$

\begin{tabular}{lclccc}
\hline & Bilabial & Apical & Velar & Uvular \\
\hline Stops & $\mathrm{p}$ & $\mathrm{t}$ & $\mathrm{k}$ & $\mathrm{q}$ \\
Voiced fricatives & $\mathrm{v}$ & $\mathrm{l}$ & $\mathrm{j}$ & $\mathrm{V}$ & $\mathrm{R}$ \\
Voiceless fricatives & & $\mathrm{d}$ & $\mathrm{s}$ & & \\
Nasals & $\mathrm{m}$ & $\mathrm{n}$ & & $\mathrm{y}$ & \\
\hline
\end{tabular}

(14) Consonant phonemes of Kolokuma Ijo (Williamson 1965) ${ }^{4}$

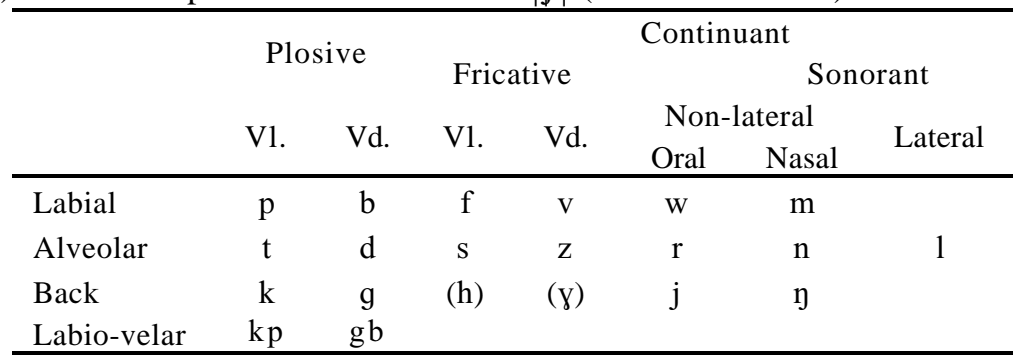

Trubetzkoy (1969, completed in 1939) does not explicitly mention the contrastive hierarchy, nor does he consistently follow it. However, many of his examples presuppose a notion of relative scope. In his discussion of the Polabian vowel 
system, for example, he explicitly refers to a hierarchy of contrasts. He writes (1969:102-103) that a "certain hierarchy existed" whereby the back $\sim$ front contrast is higher than the rounded $\sim$ unrounded one, the latter being a subclassification of the front vowels. He also proposes (1969:102) that "the maximally open vowel $a \ldots$..stood outside the classes of timbre" (i.e., the front $\sim$ back classes). This can only mean that /a/ is split off from the rest of the vowels first. The vowel system, according to Trubetzkoy's contrastive distinctions, is given in (15).

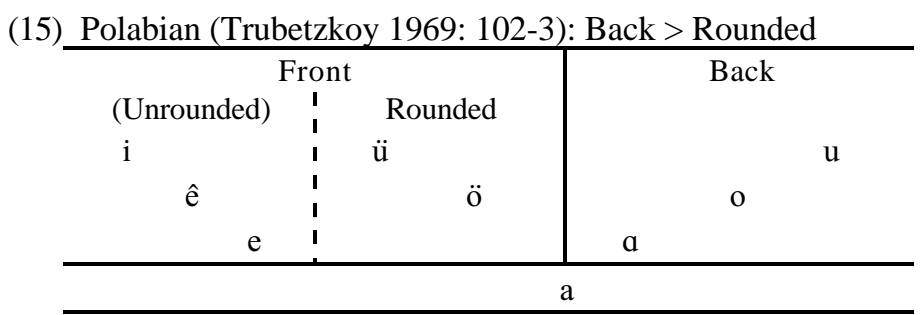

As far as I know, the first person to advocate the contrastive hierarchy was Roman Jakobson. Even he employed it inconsistently. The hierarchy was given a prominent place in Jakobson and Halle's Fundamentals of Language (1956). Jakobson and Halle (1956) refer to this hierarchy as the "dichotomous scale," and adduce "several weighty arguments" in support of this hierarchical approach to feature specification. First, they claim (1956:47) that such a system is an "optimal code" for speech participants who have to encode and decode messages.

Their second argument involves language acquisition. They suggest that distinctive features are necessarily binary because of the way they are acquired, through a series of "binary fissions". They propose (1956:41) that the order of these contrastive splits is partially fixed, thereby allowing for certain developmental sequences and ruling out others. They propose the sequence in (16) for oral resonance (primary and secondary place) features. The decimals indicate precedence relations: if one decimal sequence is entirely contained in another sequence, then the contrast corresponding to the former must precede the acquisition of the latter contrast. No implicational relations exist between features represented by an equal number of decimals. Thus, the chart in (16) is equivalent to a branching tree diagram, or a partially ordered lattice, of the sort that follows from the Successive Division Algorithm. 


\section{B. Elan Dresher}

(16) Predicted acquisition sequences (Jakobson and Halle 1956:41) Consonants: dental vs. labial

Vowels: narrow vs. wide

Narrow vowels: palatal vs. velar

Wide vowels: palatal vs. velar

Narrow palatal vowels: rounded vs. unrounded

0.1112

Wide palatal vowels: rounded vs.

$$
\text { unrounded }
$$

Velar vowels: unrounded vs. rounded

Consonants: velopalatal vs. labial and dental

Consonants: palatal vs. velar

Consonants: rounded vs. unrounded or pharyngealized vs. non-pharyngealized

Consonants: palatalized vs. non-palatalized
.... 0.112

.... 0.1121

.... 0.1122

.... 0.1123

The notion of a developmental sequence of phoneme acquisition did take hold in the field of child language. This literature, however, remained isolated from the mainstream of theoretical phonology.

Halle employed the contrastive hierarchy in The Sound Pattern of Russian (1959), though he did not emphasize its empirical nature. Also, he did not consider whether the representations he derived were the ones that best fit the patterning of the phonology, nor did he discuss the reasons for his choice of hierarchy. Rather, he argued more generally that the hierarchy is required to ensure that segments are distinct, that is, properly in contrast. Halle (1959:32) formulates a requirement that phonemes meet the Distinctness Condition (17).

(17) Distinctness of phonemes (Halle 1959:32)

Segment-type $\{\mathrm{A}\}$ will be said to be different from segment-type $\{\mathrm{B}\}$, if and only if at least one feature which is phonemic in both, has a different value in $\{A\}$ than in $\{B\}$; i.e., plus in the former and minus in the latter, or vice versa.

He gives the examples in (18). 
(18) Examples of distinctness and non-distinctness (Halle 1959:32)

a. $\quad\{\mathrm{A}\}$ is not "different from" $\{\mathrm{C}\}$

$\begin{array}{cccc} & \{\mathrm{A}\} & \{\mathrm{B}\} & \{\mathrm{C}\} \\ \text { Feature } 1 & + & - & + \\ \text { Feature 2 } & 0 & + & -\end{array}$

b. All three segment-types are "different"

$\{\mathrm{A}\} \quad\{\mathrm{B}\} \quad\{\mathrm{C}\}$

Feature $1+{ }_{-} \quad-\quad-$

$\begin{array}{llll}\text { Feature } 2 & 0 & + & -\end{array}$

(17) and (18) are essentially the argument I gave at the outset. For two items to be in contrast, they must be distinct in at least one feature. The lack of a specification does not make a segment distinct in an equipollent system, which Halle (1959) assumes. Halle (1959) argues that only a "branching diagram", i.e., a contrastive hierarchy, can ensure that the Distinctness Condition is met.

The use of "branching diagrams" was challenged by Stanley (1967), as part of his famous article arguing against unspecified features in the phonology. With respect to branching diagrams, he argued first that the Distinctness Condition is both too strong and too weak. It is too weak because it does not prevent "specious simplifications" of rules obtained by improper use of zeros. This is of course part of Stanley's general argument against zeros in the phonology, and I cannot address that here. The Distinctness Condition is too strong, in Stanley's view, because it prevents leaving out redundant features when feature values of a higher-order feature are predictable from the values of lower-order features (what we can call "underspecification from below").

For example, suppose we have a feature hierarchy as in (19a). If a segment is $\left[+\mathrm{f}_{1}\right]$, then it must be $\left[-\mathrm{f}_{2}\right]$; since this specification is not contrastive, our algorithm would omit it. For clarity, I indicate it here in parentheses. This value of $\mathrm{f}_{2}$ can be filled in by the redundancy rule (19bi). However, it is now also true that if a segment is $\left[+\mathrm{f}_{2}\right]$ then it must also be $\left[-\mathrm{f}_{1}\right]$; Stanley argues that this fact, expressed by rule (19bii), should allow us to underspecify two segments that contrast with respect to $f_{1}$ as in $(19 \mathrm{c})$. 


\section{B. Elan Dresher}

(19) Distinctness Condition and redundancies from below (Stanley 1967)

a. $\mathrm{f}_{1}>\mathrm{f}_{2}$

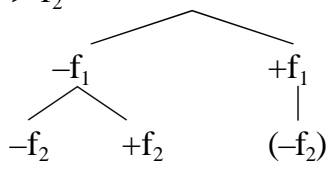

b. Redundancy rules

i. $\left[+\mathrm{f}_{1}\right] \rightarrow\left[-\mathrm{f}_{2}\right]$

c. Lexical entries violating the DC

$\begin{array}{lll} & \text { A } & \text { B } \\ \mathrm{f}_{1} & + & \\ \mathrm{f}_{2} & & +\end{array}$

ii. $\left[+\mathrm{f}_{2}\right] \rightarrow\left[-\mathrm{f}_{1}\right]$

d. Full specification

$\begin{array}{lll} & \text { A } & \text { B } \\ \mathrm{f}_{1} & + & - \\ \mathrm{f}_{2} & - & +\end{array}$

Such situations occur frequently in languages. A typical case is a language where all sonorants are voiced; then, a specification of [-voiced] necessarily implies [-sonorant]. Stanley argues that the lexical entries in (c) should be permitted, because the redundancy rules in (b) are legal and lead to the two segments being completely distinguished (d).

Stanley's argument would go through if it were indeed a requirement of the theory that all logical redundancies be expressed by zero specifications. But we have seen before that it is neither necessary nor possible for a theory to express all logical redundancies. If we take the contrastive hierarchy as the fundamental way to express contrasts in a system, then a certain amount of underspecification will follow from it. However, underspecification, on this view, is a consequence of establishing contrasts, not an end in itself. We would thus rule out specification from below as in rule (19bii): lower-order features cannot supply values for higher-order features.

Stanley's second argument against the branching trees is that it is "somewhat strange" to capture the feature hierarchy in a branching diagram because many different such diagrams can be constructed for a given set of phonemes. Stanley is correct that one must have evidence to support any proposed hierarchies. He even suggests (1967:408) where such evidence could possibly be found, "perhaps...in terms of the different ways in which different features behave in the P[honological] rules or the M[orpheme]S[tructure] rules." At the time he did not think he had such evidence, and indeed it was not (and is still not!) entirely clear what would constitute 
such evidence. However, we have seen that from the beginnings of distinctive feature theory phonologists, whether in theory or in practice, have found reasons to assign at least partial feature hierarchies.

In sum, Stanley's arguments against the branching trees, and hence against the contrastive hierarchy, rest mainly on logical, not empirical, criteria. The logical points are correct as far as they go, however his main empirical assumptions have become less relevant as phonological theory developed. Nevertheless, his arguments carried the day, and branching trees disappeared from generative phonology for the rest of the century.

However, the intuition that, as Stanley put it (408), "there is obviously some kind of hierarchical relationship among the features which must somehow be captured in the theory", continued to haunt generative phonological theory, and took a number of different forms. Three of them coexisted uneasily at the same time at the heart of mainstream generative phonology: markedness theory, underspecification theory, and feature geometry. All bear interesting, though seldom discussed, affinities with the contrastive hierarchy.

\section{Contrast in Manchu vowel systems}

I will conclude with a more recent example of an analysis that makes use of the contrastive hierarchy (see Dresher and Zhang. 2000 for a more detailed account). Zhang (1996) argues that contrastive specification following the contrastive hierarchy provides an illuminating account of the vowel system of Written Manchu, as well as of the evolution of the later Manchu languages.

Written Manchu has the vowel system in (20).

(20) Written Manchu (Zhang 1996)

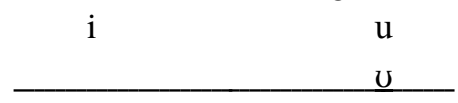

$\partial$ 


\section{B. Elan Dresher}

Vowels are divided into co-occurring sets based on ATR harmony (Zhang 1996, Zhang and Dresher 2000): / / / and /u/ spread ATR to / / and /a/. /i/ is neutral: it occurs with both sets of vowels. Even though it is phonetically ATR, it does not trigger ATR harmony. Why is that? The answer is that ATR has narrow scope in Written Manchu. If the contrastive hierarchy is as in (21), then /i/ is contrastively Coronal, but does not receive a specification for ATR.

(21) Written Manchu vowel contrasts: Low > Coronal > Labial> ATR

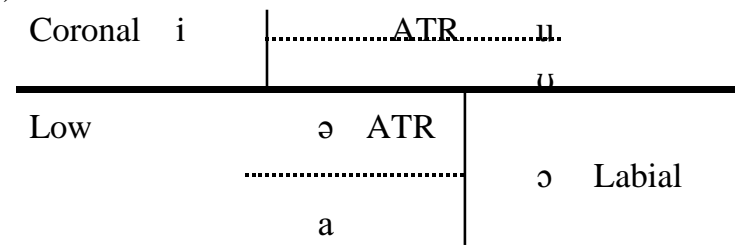

On this contrastive hierarchy, ATR is a redundant feature for /i/, filled in by the rule in (22).

(22) /i/ becomes ATR phonetically

$$
\text { [Coronal] } \rightarrow \text { ATR }
$$

Once /i/ is specified Coronal, there is no need to specify the back high vowels as Labial. In the Low region, /a/ is too far back to be Coronal, so /o/ must be Labial. These specifications accord with the patterning of labial harmony, which is triggered only by the Low round vowel. /u/ and / $/$ do not trigger labial harmony (see Zhang and Dresher 1996 for further details on labial harmony). The contrastive vowel features of Written Manchu are thus as in (23).

(23) Feature matrix for Written Manchu vowels

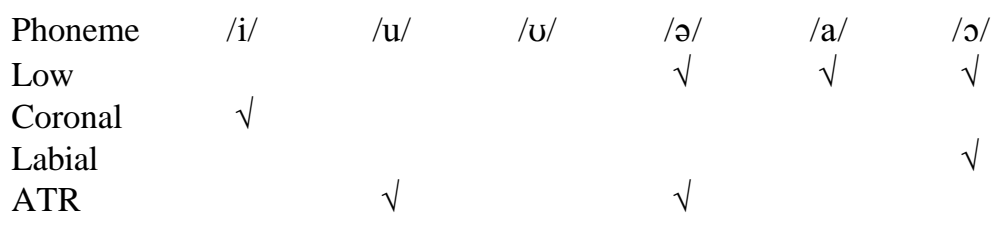


Diachronic developments support this analysis. Spoken Manchu, a descendant of Written Manchu (or of a language closely related to Written Manchu) has the vowel system shown in (24).

(24) Spoken Manchu (based on Zhao 1989, Ji et al. 1989)

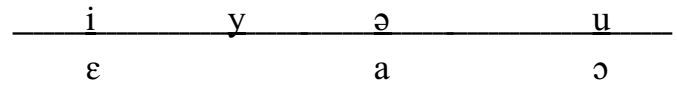

The differences between the vowel systems of Written Manchu and Spoken Manchu are summarized in (25).

(25) Differences between WM and SM vowel systems

a. Spoken Manchu no longer has a contrast between $/ \mathrm{u} /$ and $/ \mathrm{u} /$

b. In Spoken Manchu /a/ is a non-low vowel, in Written Manchu it is a low ATR counterpart to $/ \mathrm{a} /$.

c. Spoken Manchu has added coronal phonemes $/ y /$ and $/ \varepsilon /$.

These differences are all connected. It appears that Spoken Manchu first lost the distinction between $/ \mathrm{u} /$ and $/ \mathrm{v} /$. This distinction was already neutralized at the surface in many environments in Written Manchu, and in Spoken Manchu the neutralization was completed. Consequently, the ATR contrast would have rested only on /a/ and /ə/. But this contrast could now be reinterpreted as one of height, so that $/ \partial /$, of ambiguous height, was reclassified as a non-low vowel, as in (26).

(26) Spoken Manchu after loss of /v/

\begin{tabular}{c|c|c} 
Coronal i & $\partial$ & $\mathrm{u}$ \\
\hline Low & $\mathrm{a}$ & $\mathrm{o}$
\end{tabular}

Now an additional contrast has to be made among the non-low vowels: /i/ is Coronal, and $/ \mathrm{u} /$ becomes Labial to be distinguished from $/ \mathrm{\partial} /$. Evidence that $/ \mathrm{u} /$ has taken on a Labial specification is provided by the development of a new phoneme $/ y /$, a front rounded vowel created from combining /i/ and / $\mathrm{u} /(27)$. The rounding could only come from $/ \mathrm{u} /$. 


\section{B. Elan Dresher}

(27) Creation of /y/ from /u/ - /i/ and /i/ - /u/

a.

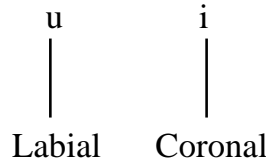

b.

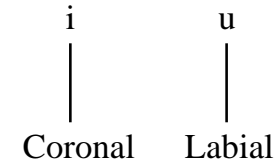

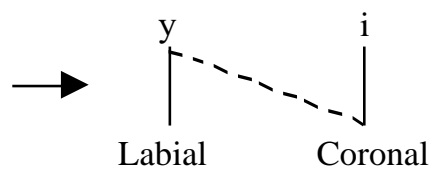

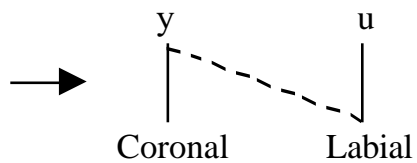

Further evidence comes from Xibe, another descendent of Written Manchu, whose vowel system is shown in (28).

(28) Xibe (Sibo) (based on Li and Zhong 1986)

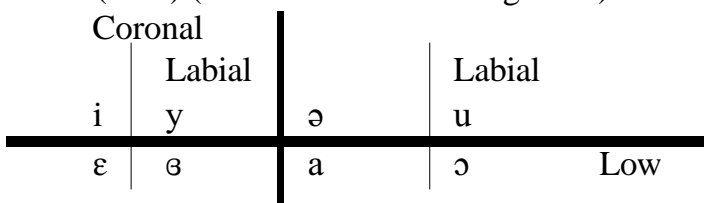

As in Spoken Manchu, Xibe / $\mathrm{u}$ / has acquired a Labial specification. Further support for this analysis comes from the operation of labial harmony in Xibe, which is triggered by $/ \mathrm{u} /$. Recall that $/ \mathrm{u} /$ in Written Manchu did not trigger labial harmony.

\section{Conclusion}

If the approach to contrastive specification sketched above is correct, it remains to be seen to what extent particular contrastive hierarchies are universal, and to what extent they can vary. This topic is the subject of ongoing work in the project on Contrast and Complexity in Phonology at the University of Toronto (http://www.chass.utoronto.ca/ contrast/). Some relevant references are: Avery (1996), Avery and Rice (1989), Balcaen (1998), Causley (1999), Dresher (1998a, b), Dresher and Rice (1993), Dresher, Piggott, and Rice (1994), Dyck (1995), Ghini 
(1999), Hall (1998), Rice (1993, 1995, 1997), Rice and Avery (1995), Walker (1993), Wu (1994), Zhang (1996), and Zhou (1999).

\section{Acknowledgements}

I would like to thank the members of the project on Contrast and Complexity in Phonology in the Department of Linguistics at the University of Toronto for many kinds of help over the years. I am especially indebted to Xi Zhang for the analysis of Manchu. This research was supported in part by grants 410-96-0842 and 410-99-1309 from the Social Sciences and Humanities Research Council of Canada.

\section{Notes}

${ }^{1}$ I assume that the set of relevant distinctive features for a particular domain is given by some theory of that domain. By "new" feature I mean one that has not already been tried. Thus, the value of $\mathrm{F}$ changes every time this step reapplies (I assume some mechanism for keeping track of which features have already been tried, but do not specify it here).

${ }^{2}$ That is, if all members of $S$ have the same value of $F$, then $F$ is not contrastive in this set.

${ }^{3}$ I have simplified Dorais's $j / d j$ and $s / c h$ to $j$ and $s$, respectively. As he makes clear, these are variants of single phonemes. Dorais does not usually indicate variants in his charts, and in related dialects in which $\mathrm{j}$ has similar variants he lists only $j$. Therefore, I keep to the usual practice of representing a phoneme by one symbol.

${ }^{4}$ I substitute $j$ for Williamson's $y$. Williamson notes that Back = palatal, velar or glottal, Vl. = voiceless, and Vd. $=$ voiced. Williamson mentions that some speakers have a marginal phoneme $/ \mathrm{\gamma} /$, but she omits it from the table. I have added it because it appears to be no less marginal than $/ \mathrm{h} /$, which is included.

\section{References}

Avery, P. 1996. The representation of voicing contrasts. Doctoral dissertation, University of Toronto. 


\section{B. Elan Dresher}

Avery, P. and Rice, K. 1989. "Segment structure and coronal underspecification". Phonology 6: 179-200.

Balcaen, M. J. 1998. Neutralization and enhancement: Vowel harmony in Aka. Ms., University of Toronto.

Causley, T. 1999. Complexity and markedness in Optimality Theory. Doctoral dissertation, University of Toronto.

Dorais, L.-J. 1990. Inuit Uqausiqatigiit: Inuit Languages and Dialects. Iqaluit: Arctic College - Nunatta Campus.

Dresher, B. E. 1998a. "Child phonology, learnability, and phonological theory". In Handbook of Language Acquisition, T. Bhatia and W. C. Ritchie (eds), 299-346. New York: Academic Press.

Dresher, B. E. 1998b. On contrast and redundancy. Paper presented at the annual meeting of the Canadian Linguistic Association, Ottawa. Ms., University of Toronto.

Dresher, B. E. and van der Hulst, H. 1998. "Head-dependent asymmetries in phonology: Complexity and visibility". Phonology 15: 317-352.

Dresher, B. E. and Rice, K. 1993. "Complexity in phonological representations”. Toronto Working Papers In Linguistics 12: i-vi.

Dresher, B. E. and Zhang, X. 2000. Contrast in Manchu vowel systems. Paper presented at the 1st International Conference on Manchu-Tungus Studies (ICMTS 1), University of Bonn, August 28 - September 1, 2000.

Dresher, B. E., Piggott, G. and Rice, K. 1994. "Contrast in phonology: Overview". Toronto Working Papers In Linguistics 13: iii-xvii.

Dyck, C. 1995. Constraining the phonology-phonetics interface, with exemplification from Spanish and Italian dialects. Doctoral dissertation, University of Toronto.

Ghini, M. 1999. Asymmetries in the phonology of Miogliola. Doctoral dissertation, University of Konstanz.

Hall, D. C. 1998. Contrastive specification for voicing in Czech. Paper presented at the MOT Phonology Workshop, University of Ottawa. Ms., University of Toronto.

Halle, M. 1959. The Sound Pattern of Russian: A Linguistic and Acoustical Investigation. The Hague: Mouton. Second printing, 1971.

Jakobson, R. and Halle, M. 1956. Fundamentals of Language. The Hague: Mouton.

Ji, Y, Zhao, Z. and Bai, L. 1989. Xiandai Manyu Babaiju [Eight Hundred Sentences of the Modern Manchu]. Beijing: Zhongyang Minzu Xueyuan Chubanshe.

Li, S. and Zhong, Q. 1986. Xiboyu Jianzhi [A Brief Introduction to the Xibe Language]. Beijing: Minzu Chubanshe.

Rice, K. 1993. "A reexamination of the feature [sonorant]: The status of 'sonorant obstruents"”. Language 69: 308-344. 
Contrast and Asymmetries in Inventories 21

Rice, K. 1995. "On vowel place features". Toronto Working Papers In Linguistics 14: 73116.

Rice, K. 1997. "Japanese NC clusters and the redundancy of postnasal voicing". Linguistic Inquiry 28: 541-551.

Rice, K. and Avery, P.. 1995. "Variability in a deterministic model of language acquisition: A theory of segmental elaboration". In Phonological acquisition and phonological theory, John Archibald (ed.), 23-42. Hillsdale, NJ: Lawrence Erlbaum.

Stanley, R. 1967. "Redundancy rules in phonology". Language 43: 393-436.

Trubetzkoy, N. 1969. Principles of Phonology. Translated from Grundzüge der Phonologie (Göttingen: Vandenhoek \& Ruprecht 1958; third edition, 1962) by C. A. M. Baltaxe. Berkeley: University of California Press.

Walker, R. 1993. Contrastive specification and vowel harmony mechanisms in the Altaic languages. Forum paper (M.A. thesis), University of Toronto.

Williamson, K. 1965. A Grammar of the Kolokuma Dialect of Ijo. Cambridge: Cambridge University Press.

Wu, Y. 1994. Mandarin segmental phonology. Doctoral dissertation, University of Toronto.

Zhang, X. 1996. Vowel systems of the Manchu-Tungus languages of China. Doctoral dissertation, University of Toronto.

Zhang, X. and B. E. Dresher. 1996. "Labial harmony in Written Manchu". Saksaha: A Review of Manchu Studies 1: 13-24.

Zhang, X. and B. E. Dresher. 2000. Tongue root harmony in Written Manchu. Paper presented at the 1st International Conference on Manchu-Tungus Studies (ICMTS 1), University of Bonn, August 28 - September 1, 2000.

Zhao, J. 1989. Xiandai Manyu Yanjiu [Studies on the Modern Manchu Language]. Beijing: Minzu Chubanshe.

Zhou, H. 1999. Vowel systems in Mandarin languages. Doctoral dissertation, University of Toronto. 